\section{$\underset{\substack{\text { hommes } \\ \text { \& migrations }}}{ }$}

\section{Hommes \& migrations}

Revue française de référence sur les dynamiques

migratoires

1307 | 2014

L'Afrique qualifiée dans la mondialisation

\title{
La diaspora kurde d'Irak au Royaume-Uni
}

\section{Bahar Baser}

\section{CpenEdition \\ Journals}

\section{Édition électronique}

URL : http://journals.openedition.org/hommesmigrations/2900

DOI : 10.4000/hommesmigrations.2900

ISSN : 2262-3353

\section{Éditeur}

Musée national de l'histoire de l'immigration

\section{Édition imprimée}

Date de publication : 1 juillet 2014

Pagination : 140-143

ISBN : 978-2-919040-28-5

ISSN : $1142-852 X$

\section{Référence électronique}

Bahar Baser, «La diaspora kurde d'Irak au Royaume-Uni », Hommes \& migrations [En ligne], 1307|

2014, mis en ligne le 15 janvier 2015, consulté le 22 septembre 2020. URL : http://

journals.openedition.org/hommesmigrations/2900 ; DOI : https://doi.org/10.4000/

hommesmigrations.2900 


\title{
REPÉRAGE
}

\section{LA DIASPORA KURDE D'IRAK AU ROYAUME-UNI}

\begin{abstract}
BAHAR BASER, chercheur associé, Centre for Trust, Peace and Social Relations (CTPSR), université de Coventry, Royaume-Uni.
\end{abstract}

$\mathrm{O}^{n}$ n estime entre 2 et 3 millions le nombre de Kurdes résidant en dehors de leur terre d'origine, la plupart ayant fui les persécutions sous les différents régimes qui se sont succédé en Turquie, Iran, Irak et Syrie. Bien qu'ils forment la plus grande diaspora d'apatrides au monde, ils restent relativement peu connus. Or, dans le monde globalisé qui est le nôtre, les diasporas, en tant que protagonistes non étatiques, sont à même d'influencer les politiques de leur pays d'origine et de leur pays d'accueil. À ce titre, la diaspora kurde et sa détermination à changer l'avenir du Kurdistan et de son peuple au Moyen-Orient occupent une place centrale. Cette diaspora étant l'une des plus actives au monde, une analyse de son fonctionnement et de sa mobilisation peut aider à éclairer la situation d'autres diasporas nées de conflits comme celles des Tamouls sri-lankais et des Palestiniens.

Avant les années 1970, la migration se cantonnait essentiellement à un petit nombre d'intellectuels kurdes venus poursuivre leurs études. Aujourd'hui, la diaspora kurde au Royaume-Uni est principalement constituée de réfugiés ayant fui les persécutions et l'oppression dans leur pays d'origine. Le coup d'État en Turquie au milieu des années 1980 engendra une autre vague importante de migrations. La guerre du Golfe devait, elle aussi, entraîner un départ massif des Kurdes vers l'Europe. La campagne d'Anfal en particulier, offensive génocidaire du régime de Saddam Hussein au Kurdistan irakien, a joué un rôle important dans cet exode'. La plus grande diaspora kurde d'Europe se trouve en Allemagne, suivie par la France, les Pays-Bas, la Suisse, la Belgique, l'Autriche et la Suède. Même si la Grande-Bretagne n'est pas le premier pays d'accueil, elle abrite une communauté kurde irakienne fortement consolidée. De même, parmi la diaspora kurde, la branche irakienne est l'une des plus développées et contribue activement à la démocratisation et aux efforts de développement de la région autonome du Kurdistan irakien. Les Kurdes au Royaume-Uni vivent principalement dans la région du Grand Londres

\section{Des statistiques difficiles}

Le débat sur l'intégration en Grande-Bretagne tourne autour des minorités visibles comme les migrants caribéens ou sud-asiatiques. Alors que ces derniers, originaires des ex-colonies, gagnent en visibilité et font l'objet de statistiques publiques, ce n'est pas le cas des Turcs et des Kurdes qui demeurent moins visibles. Généralement assimilés à un sous-groupe de la catégorie "turque", les Kurdes se retrouvent doublement exclus de ces mécanismes ${ }^{2}$, d'où un manque de discernement sur la spécificité de leurs conditions. Les raisons 
Des Kurdes devant le 10, Downing Street, les bureaux du Premier ministre britannique, en compagnie d'un parlementaire anglais d'origine kurde, Nadhim Zahawi c) KRG

culturelles, politiques et historiques de leur migration (expulsions, génocides, massacres et tortures) ne sont pas suffisamment prises en compte, ce qui explique la perception erronée dont souffre la communauté3.

II existe peu de données claires sur les Kurdes d'Irak au Royaume-Uni. La plupart des Kurdes au Royaume-Uni sont originaires de Turquie. Ceux venus d'Irak constituent le deuxième groupe au sein de la communauté, suivis par ceux de Syrie et d'Iran. Le recensement de 2001 au RoyaumeUni faisait état d'environ 11200 Kurdes d'Irak. Toutefois, le nombre important de migrants en situation irrégulière arrivés après cette date rend cette estimation obsolète ${ }^{4}$.

Certains chercheurs 5 se sont intéressés au rôle que jouent les organisations kurdes basées à Londres, notamment dans le maintien de l'identité. Latis $\operatorname{Tas}^{6}$ a longuement étudié le phénomène de pluralisme juridique, à savoir la transposition de pratiques juridiques informelles kurdes dans l'espace juridictionnel britannique. Ces études contribuent à une meilleure compréhension de la diaspora kurde en Grande-Bre- tagne. Toutefois, la prédominance des Kurdes de Turquie dans cette diaspora - dont le parcours diasporique est sensiblement différent de celui des Kurdes d'Irak - accentue d'autant plus l'invisibilité des Kurdes originaires d'autres parties du Kurdistan.

\section{Une diaspora invisible mais puissante}

Des auteurs tels que van Bruinessen7 ont souligné l'impact que peut avoir l'exil sur l'identité ethnique d'un individu et sur la question du nationalisme à longue distance. Les Kurdes entretiennent un lien avec leur pays d'origine à travers des pratiques sociales, économiques et politiques. Des événements traumatiques comme le massacre de Halabja sont commémorés par la communauté. La célébration du Nouvel An kurde ou Norouz, l'organisation de cours de danses traditionnelles et de musique permettent de garder la culture kurde vivante au sein de la diaspora. Ces différents événements ne font pas qu'entretenir l'attachement et le sentiment de solidarité intracommunautaire de la première génération. Ils assurent aussi la transmission de traditions et de valeurs culturelles aux générations futures. Nombre d'associations et centres communautaires ont été ouverts par des Kurdes, renseignant sur des questions liées à l'immigration comme le logement, le travail, l'éducation et la langue. Les nouvelles technologies et les médias aident aussi à se tenir informé des événements en cours au Kurdistan.

Pour décrire la diaspora kurde au Royaume-Uni, le site Internet officiel du gouvernement régional du

3. Rapport des droits de l'homme pour les Kurdes (KHRP), "What impact does UK government legislation and policy have on the Kurdish Diaspora?", Berghof Peace Support \& Center for Just Peace and Democracy, 2011. 4. Nazand Begikhani, Aisha Gill, Kawther Ibraheem, op. cit. 5. Omer Ugurlu, "Exploring the role of the Kurdish community organizations (London) in preserving identity of the Kurdish diaspora in London", mémoire de thèse non publié, School of Social Policy, Sociology and Social Research, 2003 ; Ipek Demir, "Battling with Memleket in London. The Kurdish diaspora's engagement with Turkey", in Journal of Ethnic Migration Studies, vol. 38, $n^{\circ}$ 5, $2012, \mathrm{pp} .815-831$. 6. Latif Tas, "One state, plural options. Kurds in the UK", in Journal of Legal Pluralism and Unofficial Law, vol. 45, n 2, 2013, pp. $167-189$. 7. Martin van Bruinessen, "Transnational aspects of the Kurdish question", texte d'étude, Robert Schuman Centre for Advanced Studies, European University Institute, Florence, 2000 


\section{REPÉRAGE}

Kurdistan (KRG) parle d'une "communauté florissante" et l'encourage à s'intégrer à la société britannique tout en entretenant des liens sociaux, culturels et économiques avec le Kurdistan. La diaspora kurde est fortement politisée depuis les années 1970. À l'époque, les activistes en exil au Royaume-Uni ont adhéré immédiatement à la cause kurde. En effet, la diaspora kurde apatride étant privée de toute représentation politique officielle, les Kurdes ont eu recours à diverses formes d'actions de protestation pour faire entendre leurs voix et attirer l'attention sur la situation de leur terre d'origine. Les manifestations et les grèves de la faim durant le régime de Saddam Hussein ont eu pour résultat la mention répétée de la situation kurde dans les bulletins d'informations de la BBC. Des associations kurdes sont parvenues à collecter des fonds considérables pour venir en aide aux réfugiés. En plus des campagnes politiques, la solidarité s'est traduite par l'envoi de fonds individuels aux familles et amis touchés par la guerre au Kurdistan. On peut donc affirmer le rôle important joué par la diaspora pendant les périodes de tumulte.

Malgré la diversité des groupes et une certaine rivalité possible entre eux, il existe une volonté de rassembler les Kurdes en une même organisation et de renseigner l'opinion publique sur des questions qui touchent au Kurdistan tout entier. Les activités diasporiques sont spécifiques au pays d'accueil. Dans le cas de la Grande-Bretagne, une nouvelle dynamique, apolitique, émerge. Une campagne visant à défier le manque de reconnaissance de l'identité kurde intitulée "Faites que I'identité kurde compte !" a été soutenue par l'Association d'études et d'étudiants kurdes (KSSO). Cette campagne appelait les Kurdes du RoyaumeUni à participer au recensement de 2011 dans le but d'accorder une place à la langue et à l'identité kurdes dans les décisions politiques. Les initiatives du KSSO sont indépendantes des partis politiques. L'association se dit pan-kurde et accueille donc des membres de tout horizon. En dehors de ces plates-formes sociétales civiles, on dénombre des manifestations culturelles, comme le Festival du film kurde à Londres, qui rassemblent, dans la diversité, des Kurdes de toutes origines.

\section{L'activisme de la diaspora kurde irakienne de nos jours}

La montée du Kurdistan irakien sur la scène du Moyen-Orient débute à la fin de la guerre du Golfe en 1991, date à laquelle il devient une région autonome, et se poursuit après 2003 par son essor économique. La diaspora kurde irakienne a joué un rôle important dans ce progrès par son implication dans les sphères sociale, économique et politique. Elle fait l'objet d'un département au sein du ministère des Relations étrangères du gouvernement régional du Kurdistan. Le KRG est fortement représenté au Royaume-Uni, ce qui s'explique par les relations historiques avec l'ancienne puissance coloniale, par l'importance de la diaspora kurde irakienne dans le pays et par la place stratégique du Royaume-Uni sur l'échiquier politique mondial. Lors d'une conférence de presse, le responsable du ministère des Relations étrangères du KRG a décrit le Royaume-Uni comme un "partenaire de choix" pour le Kurdistan. Beaucoup de politiciens et de représentants de la diaspora ont souligné son rôle en 1991 et en 2003 ainsi que l'importance de son soutien dans l'atteinte des buts fixés par le KRG concernant l'avenir du Kurdistan. ${ }^{8}$

Beaucoup de Kurdes sont rentrés au Kurdistan durant la dernière décennie. Certains d'entre 
eux, diplômés des universités britanniques ou hommes d'affaires prospères, ont endossé des rôles importants dans la sphère politique et économique kurde. Ceux qui sont restés au RoyaumeUni ont adapté leurs stratégies aux nouvelles dynamiques de la région. Auparavant, ils luttaient pour faire entendre leur voix. Désormais, ils s'efforcent de promouvoir le Kurdistan, en faisant la publicité de son développement économique, et d'améliorer l'image des Kurdes aux yeux de la communauté internationale. L'activisme politique est aux mains du KRG, tandis que les activités culturelles et sociales sont du ressort des organisations et des membres de la diaspora.

Une étude des activités organisées par les membres de la diaspora et par les corps représentants révèle que les priorités sont la reconnaissance du génocide d'Anfal et l'amélioration de l'image du Kurdistan au Royaume-Uni par la mise en place de réseaux et de contacts commerciaux. Les représentants du KRG au Royaume-Uni ont organisé plusieurs événements visant à reconnaître le caractère génocidaire de la campagne d'Anfal et à sensibiliser l'opinion publique au massacre de Halabja sous le slogan "Justice pour Halabja". L'une des plus grandes victoires pour les représentants du KRG et de la diaspora kurde fut la reconnaissance officielle par le Parlement britannique du génocide d'Anfal. Les organisations kurdes se sont beaucoup investies, faisant circuler une pétition qui a recueilli 28000 signatures et a permis d'introduire le débat au Parlement. La motion attribuait non seulement la définition de génocide aux atrocités commises, mais elle exhortait l'Union européenne et les Nations Unies à se pencher plus sérieusement sur le sujet et à s'engager dans la même voie. Récemment, le 10 mars 2014, une conférence au Parlement britannique marquait le $26^{\mathrm{e}}$ anniversaire de l'attaque chimique. Parmi les participants, on retrouvait des membres de la diaspora, des survivants du génocide de Halabja et des personnalités connues de la diaspora kurde d'Irak au RoyaumeUni, tel Nadhim Zahawi, coprésident du groupe parlementaire multipartite à Westminster chargé de la région du Kurdistan. Le KRG le présente comme le "premier parlementaire britannique né au Kurdistan”. II joue le rôle de médiateur entre les cercles politiques britanniques et les représentants du KRG, ainsi que la région du Kurdistan de manière générale. Les pratiques de lobbying en Grande-Bretagne sont le reflet de la politique diasporique : un politicien arrive parfois à répondre aux demandes de milliers de manifestants par le seul biais de ses réseaux et connexions politiques. Ce genre d'initiative sensibilise l'opinion à la situation au Kurdistan irakien et suscite aussi l'intérêt de parlementaires, journalistes et chercheurs. Un grand nombre de Kurdes d'Irak au Royaume-Uni sont hautement qualifiés et capables d'offrir leur expertise au service du Kurdistan. La seconde génération, également très qualifiée et se définissant à la fois comme kurde et britannique, est plus à même de "britanniser" les démarches revendicatives en ramenant la question kurde devant les institutions britanniques, les partis politiques et les ONG. J'ai la conviction que la nouvelle génération pourra œuvrer à la reconnaissance de la "kurdicité" en tant qu'identité ethnique distincte au Royaume-Uni, et aider la communauté kurde à trouver sa place dans la mosaïque multiculturelle de la société britannique.

Dans le monde actuel, les diasporas continueront d'exister et d'exercer une influence notable en tant qu'acteurs non étatiques. La diaspora kurde caractérisée par sa structure hétérogène, son fort potentiel et sa force inépuisable dans la défense des droits kurdes pèsera sans aucun doute sur les politiques des pays où résident les Kurdes. Les première et deuxième générations conserveront leur attachement au Kurdistan et sauront adopter de nouvelles stratégies face à l'évolution politique au Moyen-Orient et en Europe. 